\title{
Container port selection factors: heterogeneity among major market players
}

\author{
Sang-Yoon Lee*, Young-Tae Chang*** and Paul Tae-Woo Lee***
}

\begin{abstract}
This study explores the main factors considered when shippers and container shipping lines select their import/export and transshipment ports. In the present study, 38 container port selection indices were chosen from the previous research and field interviews. The scores of the 38 items were collected via survey to the three major maritime/port market players: shippers, shipping lines, and container terminal operators. In order to analyze the different priorities imposed on the port selection factors by the three market players, the ANOVA method has been employed. The empirical test shows the different perceptions about port selection attributes among service suppliers and demanders. In addition, the 38 items have been categorized into seven key factors through an exploratory factor analysis. The ANOVA technique was employed again to analyze the perspective differences for the port selection factors among the market players. The results show that there are significant differences among the players assessing the importance of the three port choice factors: liners and terminal operators give more weight to 'hinterland and terminal basic conditions' than shippers; terminal operators do not take 'line operation' as seriously as carriers and shippers; the factor of 'terminal operation' is more significantly considered by liners and terminal operators than by shippers.
\end{abstract}

Keywords: shipping line, shipper, tTerminal operator, port selection, ANOVA, scheffe multiple comparison test, exploratory factor analysis

Submission Date: 10/04/2010 Revision Date: 10/13/2010

Acceptance Date: 10/13/2010

* Corresponding Author, Associate Professor, Graduate School of Logistics Inha University Incheon, Korea. Fax: 82-32-860-8236 E-mail:sylee@inha.ac.kr

** Professor, Graduate School of Logistics Inha University Incheon, Korea.

Fax: 82-32-860-8236 E-mail:ytchang@inha.ac.kr

*** Professor, Department of Logistics and Shipping Management Kainan University, Taiwan 


\section{Introduction}

Under recent competitive maritime/port market environments, port management and operational strategies should be established and implemented with a comprehensive understanding of the key factors influencing users' port selection. For this issue, a lot of research has been undertaken and various findings and implications have been brought to light. However, little research has tried to explore the heterogeneous perspectives and priorities on port choice factors among different maritime/port market players. This paper mainly aims to identify and evaluate the key determinants of port selection and to investigate market players' different perspectives regarding port choice criteria.

In order to correspond with the research objectives proposed above, the current study adopts a questionnaire survey completed by the main market players - shipping lines, shippers and terminal operators in Korea and employs two analytic techniques, which are analysis of variance (ANOVA) and exploratory factor analysis (EFA). The remainder of this paper is structured as follows. The next section handles a literature review of port selection criteria and methodologies adopted in the existing research. Section three presents the questionnaire design and responses, while section four deals with the descriptive and statistical analysis and the results. The final section draws managerial implications and conclusions.

\section{Literature Review}

There are numerous studies on port choice issue. Determining who has more influential power in the choice of the import/export and transshipment ports between carriers and shippers has been a long and controversial argument.

Traditionally, most studies on port choice models have focused on port selection made by shippers rather than by other stake-holders such as Bird and Bland (1988), Murphy and Daley (1994), Nir et al. (2003) and Tiwary et al. (2003). For instance, Tiwary et al. (2003) argue that shippers possess stronger bargaining and/or influencing power against over shipping lines and liners' service design. According to them, the organization of global strategic alliances of shipping lines has redesigned liner service routes in response to economic growth and shippers' needs. The authors analyzed port and shipping line selection criteria considered by shippers, and assumed that location of the shipper, preferences for shipping line services, and facilities offered by ports are critical factors. Their empirical research applied a discrete choice model where each shipper faces a choice of 14 alternatives based on shipping line and port combinations. The empirical results indicated that the distance of the shipper from port, distance to destination (in case of exports), distance from origin (in case of imports), port congestion, and shipping line's fleet size play important roles. 
In contrast, recently more research has examined port choice from the perspective of the shipping lines. D'Este and Meyrick (1992) argued that port selection shifted from the shipper to the carriers as the liners grew larger in their scale of operations. They take a similar view to that of Hayuth (1987), Hayuth and Fleming (1994), Lago et al. (2001), Malchow and Kanafani (2001, 2004), and Tongzon and Sawant (2007). For instance, Lago et al. (2001) examined the routes of vessels along the U.S. West Coast between 1993 and 1999 and found that carriers tended to choose the number of ports before specifying the ports. Malchow and Kanafani (2001) identified the factors affecting the port selection for U.S. export cargo liners using a multinomial logit model and found that oceanic and inland distances affect port selection negatively. In addition, adopting a revealed preference approach, Tongzon and Sawant (2007) presented port costs and range of port services to be the only significant factors in liner's port choice.

In the real container shipping industry, carriers seem to have more influential power to select ports; however, behind this carrier's port choice behavior, shippers exert a critical influence on liners' service route designs. As Malchow and Kanafani (2004) assumed, shipping lines directly select their calling ports considering shippers' requirements, and shippers indirectly realize their preference for a port by the choice of a line providing a service route passing through that port. Therefore, it would be a more balanced approach for ports and terminals to consider the perspectives of both carriers and shippers when they establish their development plans and marketing strategies. From this point of view, some studies have been interested to find if there are significant differences in valuation of port choice criteria among the major stakeholders.

Murphy et al. (1992) investigated a series of port selection criteria from the perspectives of different market players such as ports, carrier, freight forwarders, and larger and smaller shippers in U.S. They distributed a survey to those five different groups using nine port selection factors. Their empirical study indicates that port selection attributes are evaluated differently by various participants and there exist significant differences in the following five factors: large and/or odd sized freight, large volume shipments, shipment information, claims handling, and special handling. Zan (1999) built a game theoretical model to explain the interaction between port, carriers and shippers. While it was possible to explain the interaction between shippers and carriers, he was unable to explain the interaction between port and the other parties due to lack of policy data from ports.

Song and Yeo (2004) tried to identify the factors contributing to the overall competitiveness of Chinese main ports focusing on elements related to geographical location, logistics, and operational services provided by the ports. They collected 73 elements and factors for port competitiveness through a survey to 180 professionals including ship-owners, shipping company executives, shippers, terminal operators and academics and researchers. Using the analytic hierarchy process (AHP) method, the authors found that location factor plays the most significant role for a port's competitiveness. Lirn et al. (2004) applied the AHP 
method to reveal and analyze liners' transshipment port selections. They selected 47 relevant service attributes which were categorized into 4 main service criteria (i.e. physical/technical infrastructure, geographical location, terminal cost, and management/ administration) comprising 12 sub-criteria by using two rounds of delphi surveys. The results of the AHP analysis targeting 20 carriers and 20 port operators shows that both container carriers and port service providers have a similar perception about the most important service attributes for port selection; however, the weights among the sub-criteria reveal some differences between the two survey groups. The authors revealed that the five service attributes such as handling cost, proximity to main navigation routes, proximity to import/export areas, infrastructure condition, and feeder network are the most important service attributes of transshipment ports.

However, the previous studies have not provided a comprehensive understanding about heterogeneous perspectives of different market players. Some research considered very limited port choice attributes (Murphy et al., 1992) or could not present clear interactions between port service providers and customers (Zan, 1999). In addition, some research only provided an aggregated perspective combining different market players (Song and Yeo, 2004) or did not include shippers' views since transshipment port choice are more oriented to carriers' network strategies (Lirn et al., 2004). Considering the port choice arguments and insufficiency of previous research, it is very necessary for terminal operators to explore the perspective differences among shippers, shipping lines, and operators themselves in order to properly define their customers' needs and evaluate their capabilities to respond market demand.

\section{Research Methodology}

The main methodology of this paper is to survey a sample of three different market players, i.e. shippers, shipping lines, and terminal operators, and analyze the survey results by analysis of variance and exploratory factor analysis.

\subsection{Questionnaire Survey Process}

As a first step, a draft questionnaire instrument was designed by critically referring to the various port choice factors adopted in the existing studies. In the previous studies, factors influencing the selection of ports are typically categorized into, among others, port location, port characteristics, port operations, hinterland characteristics, liners characteristics and others . The initial version of the questionnaire was pre-tested about whether expressions in the form were easy for the respondents to understand and also whether any important questions were missing or not. Through the pilot survey, respondents reported that there 
were no 'not understandable questions' or 'not available/applicable questions'. However, respondents recommended the deletion of seven items, which were not relevant port selection factors since some were too comprehensive (e.g., geographical location, terminal operation) and some were duplicated with other items left on the final version (e.g., nearness to import/export regions, port congestion, service pattern, and ship capacity). In addition, political stability was deleted due to its inefficacy in measuring port competitiveness. Meanwhile, seven new items were recommended to be added in order to consider liners' fleet operation strategies (cargo balance, alliance members' calling, competitors' calling), to include the importance of value-added logistics (VAL) activities behind ports (port backup area, free trade zone), to compensate terminal handling charges (port productivity), and to test the usefulness of port marketing for cargo inducement.

The modified questionnaire, composed of 38 port selection attributes, includes general information about carriers, shippers and terminal operators before asking the factors (see Table 1). The questionnaire consisted of two parts. The first part was concerned with background information on the sampled company and the second part with major questions on a seven point Likert scale to decide on the important attributes in port selection. Under the Likert scale, ' 1 ' designatess the lowest importance while ' 7 ' represents the highest priority. In addition, the measurement scales include the 'not available/applicable' option because potential respondents may not have all the necessary knowledge to complete all items on the questionnaire.

Table 1.

Port selection factors and indices from literature review

\begin{tabular}{l|l|l}
\hline Initial Categories & \multicolumn{1}{|c}{ Attributes } & \multicolumn{1}{c}{ Sources } \\
\hline \hline \multirow{3}{*}{ Port Location } & $\begin{array}{l}\text { Accessibility to port } \\
\text { Nearness to main truck lines } \\
\text { Ocean transportation distance } \\
\text { Nearness to inland shippers }\end{array}$ & $\begin{array}{l}\text { Slack(1985), } \\
\text { Malchow \& Kanafani (2001, 2004), } \\
\text { Yeo et al. (2004), } \\
\text { Lirn et al. (2004) }\end{array}$ \\
\hline \multirow{3}{*}{ Port Characteristics } & $\begin{array}{l}\text { Port depth } \\
\text { Port infrastructure condition } \\
\text { Port reputation } \\
\text { Feeder network }\end{array}$ & $\begin{array}{l}\text { Slack(1985), } \\
\text { Bird and Bland (1988), } \\
\text { Malchow \& Kanafani (2001, 2004), } \\
\text { Lirn et al. (2004) }\end{array}$ \\
\hline
\end{tabular}




\begin{tabular}{|c|c|c|}
\hline Initial Categories & Attributes & Sources \\
\hline Port Operation & $\begin{array}{l}\text { Port tariff } \\
\text { Berth availability } \\
\text { Terminal handling charge } \\
\text { Port productivity } \\
\text { Service reliability } \\
\text { Service speed } \\
\text { Cargo handling safety } \\
\text { Capability of operating staff } \\
\text { Relationship with union } \\
\text { Level of information technology }\end{array}$ & $\begin{array}{l}\text { Slack(1985), } \\
\text { Bird and Bland (1988), } \\
\text { Murphy and Daley (1994) } \\
\text { Nir et al. (2003), } \\
\text { Lirn et al. (2004) }\end{array}$ \\
\hline $\begin{array}{l}\text { Hinterland } \\
\text { Characteristics }\end{array}$ & $\begin{array}{l}\text { Market size } \\
\text { Cargo volume } \\
\text { Nearness to hinterland } \\
\text { Intermodal network } \\
\text { Efficient connectivity } \\
\text { Cost of Intermodal transportation } \\
\text { Port backup area } \\
\text { Free trade zone }\end{array}$ & $\begin{array}{l}\text { Slack(1985), } \\
\text { Murphy and Daley (1994), } \\
\text { Malchow \& Kanafani (2001, 2004), } \\
\text { Nir et al. (2003), } \\
\text { Yeo et al. (2004), } \\
\text { Lirn et al. (2004), } \\
\text { From interview with experts }\end{array}$ \\
\hline $\begin{array}{l}\text { Liners } \\
\text { Characteristics }\end{array}$ & $\begin{array}{l}\text { Cargo balance } \\
\text { Liners' schedule reliability } \\
\text { Liners' service frequency } \\
\text { Variety of service routes } \\
\text { Competitors' calling } \\
\text { Alliance members' calling } \\
\text { Direct calling }\end{array}$ & $\begin{array}{l}\text { Malchow \& Kanafani }(2001,2004) \text {, } \\
\text { Lirn et al. (2004), } \\
\text { Yeo et al. (2004), } \\
\text { From interview with experts }\end{array}$ \\
\hline Miscellaneous & $\begin{array}{l}\text { Claim handling } \\
\text { Special requirement handling } \\
\text { Dedicated terminal } \\
\text { Port marketing }\end{array}$ & $\begin{array}{l}\mathrm{Lu}(2000), \\
\text { Lirn et al. (2004), } \\
\text { Yeo et al. (2004), } \\
\text { From interview with experts }\end{array}$ \\
\hline
\end{tabular}

The four-page survey was sent to the potential respondents of container shipping companies (86 staff), import/export manufacturing firms (128 staff) and container terminal operating companies (42 staff) in Korea via E-mail and/or Fax. Seven of the 78 returned questionnaires (34 from liners, 20 from shippers and 17 from terminal operators) were discarded since the respondents had put the same answers on all the seven-point Likert scale items. 


\subsection{Research Framework}

After the survey data were collected, a four-step research framework was designed to analyze the data, as shown in Figure 1. First, from the aggregated survey results, the preliminary step was to extract important factors based on the information from the mean values of each item. This initial step was essential in examining how importantly market players weigh each item in their port choices. The second step was to see if there was any distinction among different market players in their port choice. This information was scrutinized by dividing the survey results into shipper, shipping line, and terminal operator groups and then by statistically testing the differences among the three groups using an analysis of variance technique. Third, in order to get a parsimonious description of data structure, an exploratory factor analysis (EFA) was adopted. This is a commonly used data reduction technique to simplify complex and diverse relationships that exist among a set of observed variables (in our case, thirty eight variables) by uncovering common dimensions or factors (Dillon and Goldstein, 1984, 53). Finally, we tried to investigate the differences in terms of port choice factors among shippers, carriers and terminal operators.

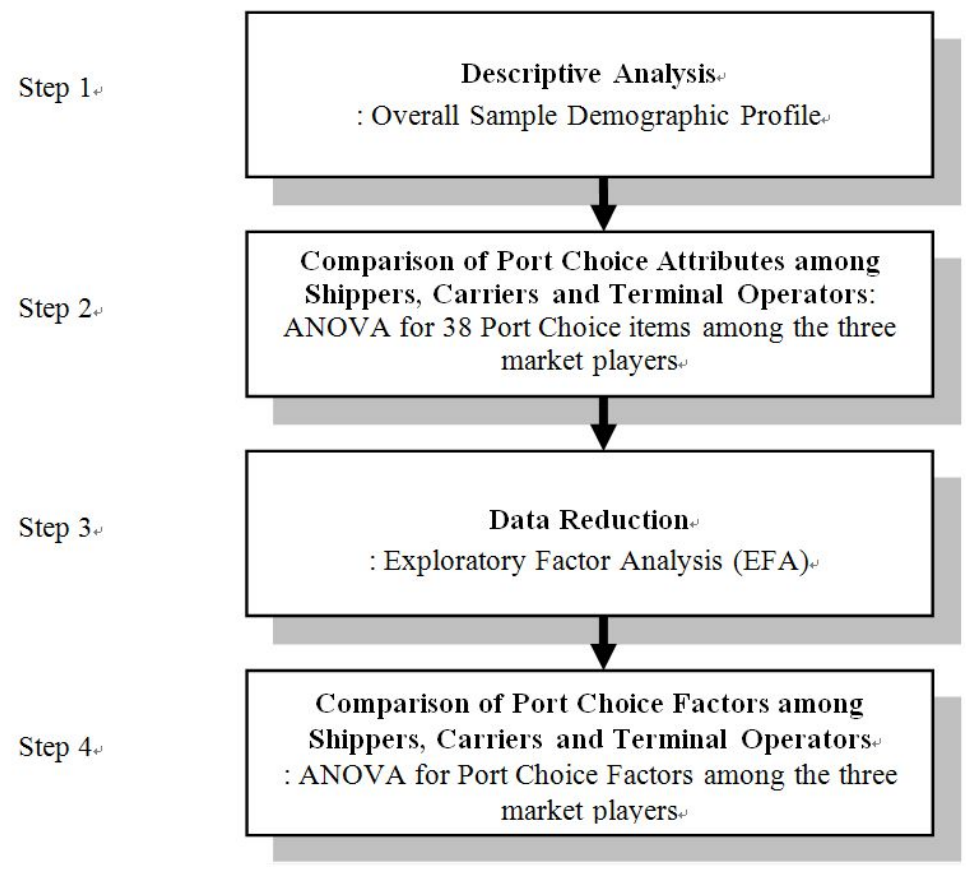

Figure 1.

Research Framework 


\section{Analysis and Results of the Survey}

\subsection{Descriptive Analysis}

The characteristics of respondents were analyzed by identifying their work experience in the industry/companies and their positions in the present firms. Firstly, the average lengths of work experience was 13.0 years in the three industries and 11.9 years in their current companies. Secondly, corresponding to the questions related to the respondents' job grade, a total of $73.2 \%$ marked their position between 15 and 20 on an ascending scale that ranged from ' 10 (clerk)' to '20 (CEO/president)', which indicates that the respondents held managerial or higher positions: the total average grades were 15.5. Considering the statistical characteristics of the respondents' positions and work experience, it was assumed that the respondents had sufficient knowledge about their firm's activities and provided accurate and reliable information.

In order to obtain company information, the respondents were asked to indicate their company age, total sales value, and number of full time employees. First, $81.7 \%$ of sampled firms had been in operation for more than 10 years and $56.3 \%$ had been operating for more than 20 years. Second, $25.4 \%$ of the firms had total sales values below 25 billion Korean Won, $46.5 \%$ of the firms recorded total sales values between 25 and 250 billion Korean Won and $28.2 \%$ firms had total sales values of over 250 billion Korean Won in $2005 .^{2}$ Thirdly, it was identified that $19.7 \%$ of the firms had 100 or fewer employees, $40.8 \%$ of the firms employed between 101 and 500 workers, $16.9 \%$ of the firms had 501 to 1,000 employees and $12.7 \%$ of the firms had more than 1,000 full time workers in 2006 .

2 USD 1 is approximately equal to KW 1,000 . 
Table 2.

General Profiles of Respondents and Respondents Companies

\begin{tabular}{|c|c|c|c|c|c|c|c|c|c|}
\hline \multicolumn{5}{|c|}{ Work experience in the Industry (years) } & \multicolumn{5}{|c|}{ Work experience in the Company (years) } \\
\hline & Liner & Shipper & Teminal & Total & & Liner & Shipper & Teminal & Total \\
\hline $1-3$ & $2.9 \%$ & $10.0 \%$ & $0.0 \%$ & $4.2 \%$ & $1-3$ & $11.8 \%$ & $10.0 \%$ & $0.0 \%$ & $8.5 \%$ \\
\hline $4-6$ & $8.8 \%$ & $10.0 \%$ & $0.0 \%$ & $7.0 \%$ & $4-6$ & $17.6 \%$ & $5.0 \%$ & $11.8 \%$ & $12.7 \%$ \\
\hline $7-9$ & $17.6 \%$ & $15.0 \%$ & $23.5 \%$ & $18.3 \%$ & $7-9$ & $2.9 \%$ & $15.0 \%$ & $11.8 \%$ & $8.5 \%$ \\
\hline $10-12$ & $23.5 \%$ & $20.0 \%$ & $17.6 \%$ & $21.1 \%$ & $10-12$ & $20.6 \%$ & $15.0 \%$ & $23.5 \%$ & $19.7 \%$ \\
\hline $13-15$ & $20.6 \%$ & $25.0 \%$ & $11.8 \%$ & $19.7 \%$ & $13-15$ & $20.6 \%$ & $20.0 \%$ & $11.8 \%$ & $18.3 \%$ \\
\hline $16-18$ & $8.8 \%$ & $5.0 \%$ & $29.4 \%$ & $12.7 \%$ & $16-18$ & $11.8 \%$ & $10.0 \%$ & $35.3 \%$ & $16.9 \%$ \\
\hline $19-21$ & $2.9 \%$ & $5.0 \%$ & $11.8 \%$ & $5.6 \%$ & $19-21$ & $2.9 \%$ & $10.0 \%$ & $0.0 \%$ & $4.2 \%$ \\
\hline $22-24$ & $8.8 \%$ & $10.0 \%$ & $0.0 \%$ & $7.0 \%$ & $22-24$ & $5.9 \%$ & $10.0 \%$ & $0.0 \%$ & $5.6 \%$ \\
\hline $25-27$ & $2.9 \%$ & $0.0 \%$ & $0.0 \%$ & $1.4 \%$ & $25-27$ & $2.9 \%$ & $0.0 \%$ & $0.0 \%$ & $1.4 \%$ \\
\hline $28-30$ & $0.0 \%$ & $0.0 \%$ & $5.9 \%$ & $1.4 \%$ & $28-30$ & $0.0 \%$ & $0.0 \%$ & $0.0 \%$ & $0.0 \%$ \\
\hline$>31$ & $2.9 \%$ & $0.0 \%$ & $0.0 \%$ & $1.4 \%$ & $>31$ & $2.9 \%$ & $0.0 \%$ & $0.0 \%$ & $1.4 \%$ \\
\hline Sum & $100.0 \%$ & $100.0 \%$ & $100.0 \%$ & $100.0 \%$ & Sum & $100.0 \%$ & $100.0 \%$ & $100.0 \%$ & $100.0 \%$ \\
\hline \multicolumn{5}{|c|}{ Respondents' position } & \multicolumn{5}{|c|}{ Company age (years) } \\
\hline & Liner & Shipper & Teminal & Total & & Liner & Shipper & Teminal & Total \\
\hline 10 & $0.0 \%$ & $10.0 \%$ & $5.9 \%$ & $4.2 \%$ & $<5$ & $0.0 \%$ & $20.0 \%$ & $0.0 \%$ & $5.6 \%$ \\
\hline 11 & $2.9 \%$ & $0.0 \%$ & $5.9 \%$ & $2.8 \%$ & $5-10$ & $8.8 \%$ & $15.0 \%$ & $17.6 \%$ & $12.7 \%$ \\
\hline 12 & $5.9 \%$ & $0.0 \%$ & $5.9 \%$ & $4.2 \%$ & $11-15$ & $5.9 \%$ & $0.0 \%$ & $29.4 \%$ & $9.9 \%$ \\
\hline 13 & $8.8 \%$ & $5.0 \%$ & $5.9 \%$ & $7.0 \%$ & $16-20$ & $2.9 \%$ & $5.0 \%$ & $52.9 \%$ & $15.5 \%$ \\
\hline 14 & $14.7 \%$ & $0.0 \%$ & $5.9 \%$ & $8.5 \%$ & $20-25$ & $2.9 \%$ & $20.0 \%$ & $0.0 \%$ & $7.0 \%$ \\
\hline 15 & $29.4 \%$ & $5.0 \%$ & $17.6 \%$ & $19.7 \%$ & $>25$ & $79.4 \%$ & $40.0 \%$ & $0.0 \%$ & $49.3 \%$ \\
\hline 16 & $17.6 \%$ & $30.0 \%$ & $0.0 \%$ & $16.9 \%$ & sum & $100.0 \%$ & $100.0 \%$ & $100.0 \%$ & $100.0 \%$ \\
\hline 17 & $11.8 \%$ & $20.0 \%$ & $29.4 \%$ & $18.3 \%$ & & & & & \\
\hline 18 & $5.9 \%$ & $20.0 \%$ & $11.8 \%$ & $11.3 \%$ & & & & & \\
\hline 19 & $0.0 \%$ & $0.0 \%$ & $11.8 \%$ & $2.8 \%$ & & & & & \\
\hline 20 & $2.9 \%$ & $10.0 \%$ & $0.0 \%$ & $4.2 \%$ & & & & & \\
\hline Sum & $100.0 \%$ & $100.0 \%$ & $100.0 \%$ & $100.0 \%$ & & & & & \\
\hline \multicolumn{5}{|c|}{ Total sales value (100 million Korean Won) } & \multicolumn{5}{|c|}{ Full time employees } \\
\hline & Liner & Shipper & Teminal & Total & & Liner & Shipper & Teminal & Total \\
\hline$<<10$ & $0.0 \%$ & $15.0 \%$ & $0.0 \%$ & $4.2 \%$ & $<100$ & $17.6 \%$ & $30.0 \%$ & $11.8 \%$ & $19.7 \%$ \\
\hline $10-50$ & $5.9 \%$ & $5.0 \%$ & $5.9 \%$ & $5.6 \%$ & $101-300$ & $32.4 \%$ & $20.0 \%$ & $23.5 \%$ & $26.8 \%$ \\
\hline $51-100$ & $11.8 \%$ & $0.0 \%$ & $5.9 \%$ & $7.0 \%$ & $301-500$ & $29.4 \%$ & $0.0 \%$ & $0.0 \%$ & $14.1 \%$ \\
\hline $101-250$ & $2.9 \%$ & $10.0 \%$ & $17.6 \%$ & $8.5 \%$ & $501-1000$ & $0.0 \%$ & $20.0 \%$ & $47.1 \%$ & $16.9 \%$ \\
\hline $251-500$ & $14.7 \%$ & $15.0 \%$ & $11.8 \%$ & $14.1 \%$ & $1001-2000$ & $5.9 \%$ & $10.0 \%$ & $17.6 \%$ & $9.9 \%$ \\
\hline $501-1000$ & $0.0 \%$ & $10.0 \%$ & $5.9 \%$ & $4.2 \%$ & $>2000$ & $14.7 \%$ & $20.0 \%$ & $0.0 \%$ & $12.7 \%$ \\
\hline $1001-2500$ & $29.4 \%$ & $5.0 \%$ & $52.9 \%$ & $28.2 \%$ & Sum & $100.0 \%$ & $100.0 \%$ & $100.0 \%$ & $100.0 \%$ \\
\hline $2501-5000$ & $11.8 \%$ & $0.0 \%$ & $0.0 \%$ & $5.6 \%$ & & & & & \\
\hline$>5000$ & $23.5 \%$ & $40.0 \%$ & $0.0 \%$ & $22.5 \%$ & & & & & \\
\hline Sum & $100.0 \%$ & $100.0 \%$ & $100.0 \%$ & $100.0 \%$ & & & & & \\
\hline
\end{tabular}




\subsection{Comparison of Port Choice Iitems among Shippers, Carriers and Terminal Operators}

Table 3 presents the heterogeneous perspectives among three market players concerning the 38 port selection factors (see appendix 1 for the mean scores for all the items). Shipping lines consider 'schedule reliability', 'accessibility to port', 'berth availability', 'terminal handling charge (THC)', 'cargo volume', 'infrastructure condition', 'liners" service frequency', 'variety of service routes', 'direct calling', and 'port productivity' as the 10 most important indices while shippers recognize 'service speed' 'schedule reliability', 'special requirement handling', 'cargo handling safety', 'nearness to inland shippers', 'service reliability', 'accessibility to port', 'problem handling', intermodal cost' and 'port tariff' as critical to port choice. In addition, terminal operators impose more weights on 'port depth', 'port productivity', 'infrastructure condition', 'cargo volume', 'berth availability', 'market size', 'nearness to main truck', 'accessibility to port', 'service speed' and 'THC'.

Table 3.

Three Market Players' Different Priority for the Port Selection Criteria

\begin{tabular}{c|l|l|l}
\hline Ranking & \multicolumn{1}{|c|}{ Liners } & \multicolumn{1}{c}{ Shippers } & \multicolumn{1}{c}{ Terminal Operators } \\
\hline \hline 1 & Liners' schedule reliability & Service speed & Port depth \\
2 & Accessibility to port & Liners' schedule reliability & Port productivity \\
3 & Berth availability & Special requirement handling & Port infrastructure condition \\
4 & Terminal handling charge & Cargo handling safety & Cargo volume \\
5 & Cargo volume & Nearness to inland shippers & Berth availability \\
6 & Port infrastructure condition & Service reliability & Market size \\
7 & Liners' Service frequency & Accessibility to port & Nearness to main truck lines \\
8 & Variety of service routes & Problem handling & Accessibility to port \\
9 & Direct calling & Intermodal cost & Service speed \\
10 & Port productivity & Port tariff & Terminal handling charge \\
\hline 11 & Service speed & Port productivity & Port tariff \\
12 & Feeder network & Liners' Service frequency & Feeder network \\
13 & Service reliability & Nearness to main truck lines & Service reliability \\
14 & Cargo balance & Berth availability & Liners' schedule reliability \\
15 & Cargo handling safety & Terminal handling charge & Information technology \\
16 & Intermodal network & Intermodal network & Liners' Service frequency \\
17 & Port tariff & Efficient intermodalism & Nearness to hinterland \\
18 & Efficient intermodalism & Nearness to hinterland & Direct calling \\
19 & Quality of staff & Port infrastructure condition & Efficient intermodalism \\
20 & Port depth & Feeder network & Special requirement handling \\
22 & Market size & Port backup area & Ocean distance \\
23 & Competitor's calling & Free trade zone & Port size \\
\hline
\end{tabular}




\begin{tabular}{|c|c|c|c|}
\hline Ranking & Liners & Shippers & Terminal Operators \\
\hline 24 & Problem handling & Competitor's calling & Nearness to inland shippers \\
\hline 25 & Nearness to main truck lines & Variety of service routes & Problem handling \\
\hline 26 & special requirement handling & Quality of staff & Port reputation \\
\hline 27 & Alliance member's calling & Cargo volume & Intermodal network \\
\hline 28 & Intermodal cost & Ocean distance & Alliance member's calling \\
\hline 29 & Port size & Dedicated terminal & Intermodal cost \\
\hline 30 & Port reputation & Port reputation & Variety of service routes \\
\hline 31 & Information technology & Alliance member's calling & Quality of staff \\
\hline 32 & Free trade zone & Market size & Competitor's calling \\
\hline 33 & Nearness to inland shippers & Port size & Port marketing \\
\hline 34 & Ocean distance & Cargo balance & Dedicated terminal \\
\hline 35 & Port backup area & Information technology & Cargo balance \\
\hline 36 & Relationship with union & Port depth & Port backup area \\
\hline 37 & Port marketing & Relationship with union & Relationship with union \\
\hline 38 & Dedicated terminal & Port marketing & Free trade zone \\
\hline
\end{tabular}

'Accessibility to port' is the only common critical port selection item. An interesting point is that ocean transportation service providers (i.e. liners and terminal operators) present similar importance on the items in the top 10 criteria, while service demanders show a different perspective for the criteria. Meanwhile, the uncritical items indicated by the three market players show somewhat different perspectives. They do not commonly consider 'relationship with union,' 'port marketing' and 'dedicated terminal' important factors in port choice. An interesting finding is that shippers and terminal operators point out that 'cargo balance' is not a considerable factor, while shipping companies recognize it as a very important factor.

Table 4.

Scheffe Multiple Comparison Test for 38 items

\begin{tabular}{|c|c|c|c|c|c|c|c|}
\hline \multirow{2}{*}{ Factor } & \multicolumn{3}{|c|}{ Level of Significance } & \multirow{2}{*}{ Factor } & \multicolumn{3}{|c|}{ Level of Significance } \\
\hline & \multicolumn{3}{|c|}{ Shippers } & & & Shippers & erminals \\
\hline \multirow{2}{*}{$\begin{array}{l}\text { Accessibility } \\
\text { to port }\end{array}$} & Liners & \multirow[t]{2}{*}{0.3667} & 0.3424 & \multirow{2}{*}{ Market size } & Liners & $0.0385^{*}$ & 0.7984 \\
\hline & Shippers & & 0.9940 & & Shippers & & $0.0213^{*}$ \\
\hline \multirow{2}{*}{$\begin{array}{l}\text { Nearness to } \\
\text { truck }\end{array}$} & Liners & \multirow[t]{2}{*}{0.9677} & 0.8134 & \multirow{2}{*}{$\begin{array}{c}\text { Cargo } \\
\text { volume }\end{array}$} & Liners & $0.0013^{*}$ & 0.8823 \\
\hline & Shippers & & 0.9371 & & Shippers & & $0.0201 *$ \\
\hline \multirow{2}{*}{$\begin{array}{c}\text { Ocean } \\
\text { distance }\end{array}$} & Liners & \multirow[t]{2}{*}{0.9858} & 0.7846 & \multirow{2}{*}{$\begin{array}{c}\text { Nearness to } \\
\text { hinterland }\end{array}$} & Liners & 0.5184 & 0.6193 \\
\hline & Shippers & & 0.8898 & & Shippers & & 0.9934 \\
\hline \multirow{2}{*}{$\begin{array}{l}\text { Nearness to } \\
\text { shippers }\end{array}$} & Liners & \multirow[t]{2}{*}{0.0613} & 0.9511 & \multirow{2}{*}{$\begin{array}{c}\text { Intermodal } \\
\text { network }\end{array}$} & Liners & \multirow[t]{2}{*}{0.4081} & 0.0645 \\
\hline & Shippers & & 0.2142 & & Shippers & & 0.6268 \\
\hline
\end{tabular}




\begin{tabular}{|c|c|c|c|c|c|c|c|}
\hline \multirow{2}{*}{ Factor } & \multicolumn{3}{|c|}{ Level of Significance } & \multirow{2}{*}{ Factor } & \multicolumn{3}{|c|}{ Level of Significance } \\
\hline & & Shippers & erminals & & & Shippers & erminals \\
\hline \multirow{2}{*}{ Port depth } & Liners & 0.0619 & 0.3902 & \multirow{2}{*}{$\begin{array}{c}\text { Efficient } \\
\text { intermodalism }\end{array}$} & \multirow{2}{*}{\multicolumn{2}{|c|}{0.5592}} & 0.3473 \\
\hline & Shippers & & $0.0064^{*}$ & & & & 0.9321 \\
\hline \multirow{2}{*}{$\begin{array}{c}\text { Port } \\
\text { infrastructure }\end{array}$} & Liners & $0.0440 *$ & 0.9846 & \multirow{2}{*}{$\begin{array}{c}\text { Intermodal } \\
\text { cost }\end{array}$} & \multirow{2}{*}{\multicolumn{2}{|c|}{0.5482}} & 0.5304 \\
\hline & Shippers & & 0.1363 & & & & 0.1568 \\
\hline \multirow{2}{*}{ Port size } & Liners & 0.2564 & 0.9433 & \multirow{2}{*}{$\begin{array}{c}\text { Cargo } \\
\text { balance }\end{array}$} & Liners & \multirow[t]{2}{*}{$0.0237^{*}$} & $0.0123^{*}$ \\
\hline & Shippers & & 0.5396 & & Shippers & & 0.9522 \\
\hline \multirow{2}{*}{$\begin{array}{c}\text { Port } \\
\text { reputation }\end{array}$} & Liners & 0.3954 & 0.8181 & \multirow{2}{*}{$\begin{array}{c}\text { Port backup } \\
\text { area }\end{array}$} & Liners & \multirow[t]{2}{*}{0.7352} & 0.5436 \\
\hline & Shippers & & 0.8442 & & Shippers & & 0.2551 \\
\hline \multirow{2}{*}{$\begin{array}{c}\text { Feeder } \\
\text { network }\end{array}$} & Liners & 0.1114 & 0.5095 & \multirow{2}{*}{$\begin{array}{l}\text { Free trade } \\
\text { zone }\end{array}$} & Liners & \multirow[t]{2}{*}{0.9992} & 0.1423 \\
\hline & Shippers & & 0.7456 & & Shippers & & 0.1963 \\
\hline \multirow{2}{*}{ Port tariff } & Liners & 0.9691 & 0.9196 & \multirow{2}{*}{$\begin{array}{c}\text { Liner's } \\
\text { reliability }\end{array}$} & Liners & \multirow[t]{2}{*}{0.8562} & 0.0684 \\
\hline & Shippers & & 0.9881 & & Shippers & & 0.2629 \\
\hline \multirow{2}{*}{$\begin{array}{c}\text { Berth } \\
\text { availability }\end{array}$} & Liners & $0.0383^{*}$ & 0.6867 & \multirow{2}{*}{$\begin{array}{c}\text { Liner's } \\
\text { frequency }\end{array}$} & Liners & \multirow[t]{2}{*}{0.5267} & 0.2396 \\
\hline & Shippers & & 0.3538 & & Shippers & & 0.8521 \\
\hline \multirow{2}{*}{ THC } & Liners & 0.0678 & 0.2667 & \multirow{2}{*}{$\begin{array}{c}\text { Variety of } \\
\text { routes }\end{array}$} & Liners & \multirow{2}{*}{$0.0425^{*}$} & $0.0389^{*}$ \\
\hline & Shippers & & 0.8611 & & Shippers & & 0.9934 \\
\hline \multirow{2}{*}{$\begin{array}{c}\text { Port } \\
\text { productivity }\end{array}$} & Liners & 0.5027 & 0.9825 & Competitor & Liners & 0.4085 & 0.2274 \\
\hline & Shippers & & 0.5042 & calling & Shippers & & 0.9259 \\
\hline Service & Liners & 0.9151 & 0.3971 & Alliance calling & Liners & 0.3492 & 0.7015 \\
\hline reliability & Shippers & & 0.6852 & Amlance calling & Shippers & & 0.8813 \\
\hline Service & Liners & 0.8135 & 0.6700 & & Liners & 0.0825 & 0.1772 \\
\hline speed & Shippers & & 0.4026 & Direct calling & Shippers & & 0.9541 \\
\hline Handling & Liners & 0.9897 & 0.2068 & & Liners & 0.8499 & 0.4299 \\
\hline safety & Shippers & & 0.2272 & Problem nandling & Shippers & & 0.2570 \\
\hline Quality & Liners & 0.1172 & 0.0754 & Special & Liners & 0.3111 & 0.8001 \\
\hline of staff & Shippers & & 0.9587 & requirement & Shippers & & 0.1664 \\
\hline Relationship & Liners & 0.8633 & 0.8282 & & Liners & 0.7828 & 0.9709 \\
\hline with union & Shippers & & 0.9960 & Dedicated terminal & Shippers & & 0.9257 \\
\hline & Liners & 0.1512 & 0.9049 & & Liners & 0.7983 & 0.7896 \\
\hline 11 & Shippers & & 0.1212 & Port marketing & Shippers & & 0.4965 \\
\hline
\end{tabular}

* Mean differences are significant at $\mathrm{P}<0.05$

Table 3 presents different overall perspectives among shipping lines, shippers and terminal operating companies (about 38 port selection criteria). However, it only presents descriptive differences but not statistically significant heterogeneous among the players . In order to assess whether the means of the 38 factors were significantly different across the three market players, the one-way analysis of variance (ANOVA) was adopted. The results of the Scheffe multiple comparison test are shown in Table 4. 
The results show statistical differences of port selection perspective among the players in the items of 'port depth,' 'port infrastructure,' 'berth availability,' 'market size,' 'cargo volume,' 'cargo balance' and 'variety of service routs'. Concerning 'port depth,' liners and terminal operators have no significant difference but shippers impose less weight compared to the terminal operators. In the cases of 'port infrastructure' and 'berth availability' liners and shippers show significant differences; compared to shippers, liners consider 'port infrastructure condition' and 'berth availability' as significant attributes for port selection. Regarding 'market size' and 'cargo volume' shipping firms and terminal operating companies have similar perspectives but possess significantly higher priorities to shippers. Meanwhile, liners place a lot of importance on 'cargo balance' and 'variety of service routes' compared to shippers and terminal operators (see Appendix 1).

\subsection{Data Reduction: Exploratory Factor Analysis}

To interpret the survey results more clearly, we tried to reduce the dimensionality and burden of interpretation of the numerous variables and responses. Principal component analysis, a type of factor analysis, transforms the original set of variables into a smaller set of linear combinations that account for most of the variances of the original set. The purpose of principal component analysis is to determine factors in order to explain as much of the total variation in the data as possible with as few of these factors as possible (Dillon and Goldstein, 1984, 23-28).

Table 5 reveals several important factors or principal components. Although thirty-eight variables were specified in the questionnaire, it is shown that the dimensionality can be reduced into a smaller number of factors (principal components). Since there can be the same number of principal components as the number of variables in principal component analysis, one should decide how many components to retain. Unfortunately, there is no universally accepted method for doing so, and the decision becomes largely judgmental and a matter of taste. One commonly used method is the "root of greater than one" criterion approach, which is to retain those components whose eigen values are greater than one (Dillon and Goldstein, 1984, 23-52).

Grouping the variables into the factors should totally depend upon the value of loadings. Therefore, each variable's loading with the largest absolute value in its respective row across the factors should be grouped into the respective factors as shown as shaded italic bold-faced variables in the table. The factor solution resulted in seven factors with eigen values greater than unity, which are 'customer service capability', 'advanced port management', 'hinterland and terminal basic condition', 'shipping line operation', 'terminal operation', 'transportation distance', and 'intermodal system'. The seven factor solutions for the 38 items accounted for $75.9 \%$ of the variance. The results of a reliability test show that the derived factors are reliable. 
Table 5.

Exploratory Factor Analysis Result

\begin{tabular}{|c|c|c|c|c|c|c|c|c|}
\hline $\begin{array}{c}\text { Factor } \\
\text { (Cronbach's Alpha) }\end{array}$ & Attributes & 1 & 2 & 3 & 4 & 5 & 6 & 7 \\
\hline \multirow{7}{*}{$\begin{array}{l}\text { Customer service } \\
\text { capability } \\
(0.917)\end{array}$} & Problem handling & 0.788 & 0.211 & -0.046 & 0.244 & 0.066 & 0.002 & 0.327 \\
\hline & Cargo handling safety & 0.786 & 0.261 & 0.161 & 0.211 & 0.114 & 0.181 & 0.032 \\
\hline & Service speed & 0.784 & 0.038 & 0.152 & 0.215 & 0.206 & 0.214 & 0.135 \\
\hline & Service reliability & 0.780 & 0.121 & 0.272 & 0.071 & 0.204 & -0.037 & 0.158 \\
\hline & Special requirement handling & 0.588 & 0.286 & -0.155 & 0.188 & 0.086 & 0.095 & 0.460 \\
\hline & Intermodal cost & 0.543 & 0.385 & 0.203 & 0.237 & 0.032 & 0.342 & 0.315 \\
\hline & Port productivity & 0.532 & 0.113 & 0.477 & 0.186 & 0.377 & 0.085 & -0.032 \\
\hline \multirow{7}{*}{$\begin{array}{l}\text { Advanced port } \\
\text { management } \\
(0.894)\end{array}$} & Relationship with union & 0.137 & 0.821 & 0.196 & 0.046 & 0.100 & 0.037 & 0.116 \\
\hline & Port backup area & 0.152 & 0.773 & -0.088 & 0.285 & 0.026 & 0.235 & 0.146 \\
\hline & Information technology & 0.127 & 0.738 & 0.315 & 0.103 & 0.253 & -0.124 & 0.000 \\
\hline & Free trade zone & 0.333 & 0.708 & -0.062 & 0.337 & 0.102 & -0.013 & 0.245 \\
\hline & Port reputation & 0.061 & 0.573 & 0.071 & 0.178 & 0.491 & -0.086 & 0.036 \\
\hline & Dedicated terminal & 0.116 & 0.561 & 0.283 & 0.051 & -0.082 & 0.231 & 0.550 \\
\hline & Quality of staff & 0.486 & 0.506 & 0.235 & 0.117 & 0.332 & -0.014 & 0.077 \\
\hline \multirow{6}{*}{$\begin{array}{c}\text { Hinterland and terminal } \\
\text { basic condition } \\
(0.876)\end{array}$} & Cargo volume & 0.151 & 0.139 & 0.848 & 0.152 & 0.180 & 0.104 & 0.229 \\
\hline & Market size & 0.091 & 0.208 & 0.797 & 0.126 & 0.082 & 0.084 & 0.175 \\
\hline & Port depth & -0.006 & 0.037 & 0.697 & 0.052 & 0.391 & 0.362 & 0.007 \\
\hline & Nearness to hinterland & 0.314 & 0.171 & 0.581 & 0.179 & 0.062 & 0.195 & 0.467 \\
\hline & Terminal handling charge & 0.295 & 0.272 & 0.488 & 0.413 & 0.353 & 0.197 & -0.232 \\
\hline & Berth availability & 0.309 & 0.327 & 0.447 & 0.302 & 0.447 & 0.133 & -0.097 \\
\hline \multirow{7}{*}{$\begin{array}{l}\text { Shipping line operation } \\
\qquad(0.936)\end{array}$} & Liners' schedule reliability & 0.526 & 0.110 & 0.031 & 0.727 & 0.000 & 0.053 & 0.032 \\
\hline & Direct calling & 0.189 & 0.235 & 0.211 & 0.688 & 0.191 & -0.146 & 0.423 \\
\hline & Cargo balance & 0.214 & 0.518 & 0.188 & 0.640 & 0.131 & 0.076 & 0.099 \\
\hline & Liners' Service frequency & 0.553 & 0.237 & 0.178 & 0.600 & 0.205 & 0.095 & 0.167 \\
\hline & Alliance member's calling & 0.137 & 0.531 & 0.205 & 0.563 & 0.161 & 0.071 & 0.232 \\
\hline & Competitor's calling & 0.200 & 0.446 & 0.244 & 0.511 & 0.269 & 0.119 & 0.146 \\
\hline & Variety of service routes & 0.502 & 0.331 & 0.243 & 0.508 & 0.345 & -0.014 & -0.019 \\
\hline \multirow{5}{*}{$\begin{array}{c}\text { Terminal operation } \\
(0.865)\end{array}$} & Port tariff & 0.112 & 0.165 & 0.033 & 0.295 & 0.703 & 0.357 & 0.049 \\
\hline & Port size & 0.271 & 0.278 & 0.314 & -0.214 & 0.683 & -0.105 & 0.069 \\
\hline & Port infrastructure & 0.273 & 0.021 & 0.410 & 0.181 & 0.613 & 0.005 & 0.300 \\
\hline & Feeder network & 0.161 & 0.303 & 0.269 & 0.247 & 0.578 & 0.005 & 0.160 \\
\hline & Accessibility to port & 0.307 & -0.058 & 0.124 & 0.420 & 0.506 & 0.340 & 0.381 \\
\hline \multirow{3}{*}{$\begin{array}{l}\text { Transportation distance } \\
\qquad(0.783)\end{array}$} & Ocean transportation distance & -0.157 & 0.123 & 0.065 & 0.041 & 0.220 & 0.796 & 0.147 \\
\hline & Nearness to inland shippers & 0.351 & -0.023 & 0.113 & 0.027 & -0.219 & 0.788 & 0.104 \\
\hline & Nearness to main truck lines & 0.275 & -0.023 & 0.409 & -0.012 & 0.138 & 0.749 & -0.007 \\
\hline \multirow{3}{*}{$\begin{array}{l}\text { Intermodal system } \\
(0.811)\end{array}$} & Efficient intermodalism & 0.342 & 0.128 & 0.255 & 0.207 & 0.116 & 0.266 & 0.587 \\
\hline & Port marketing & 0.230 & 0.406 & 0.159 & 0.059 & 0.296 & -0.004 & 0.548 \\
\hline & Intermodal network & 0.453 & 0.196 & 0.273 & 0.359 & 0.195 & 0.117 & 0.506 \\
\hline
\end{tabular}

Note: Kaiser-Meyer-Olkin Measure of Sampling Adequacy is 0.775

In order to assess whether the means of the seven factors were significantly different across the three market players, the analysis of variance (ANOVA) technique was adopted again. The results of the Scheffe multiple comparison test are shown in Table 6. Appendix 
2 presents the means and standard deviations of the seven port selection factors evaluated by each market player.

Table 6.

Scheffe Multiple Comparison Test for Seven Port Selection Factors

\begin{tabular}{|c|c|c|c|}
\hline \multirow{2}{*}{ Factor } & \multicolumn{3}{|c|}{ Level of Significance } \\
\hline & & Shippers & Terminals \\
\hline Customer Service Capability & $\begin{array}{l}\text { Liners } \\
\text { Shippers }\end{array}$ & 0.945 & $\begin{array}{l}0.421 \\
0.340\end{array}$ \\
\hline Advanced Port Management & $\begin{array}{l}\text { Liners } \\
\text { Shippers }\end{array}$ & 0.479 & $\begin{array}{l}0.180 \\
0.811\end{array}$ \\
\hline Hinterland and Terminal Basic Condition & $\begin{array}{l}\text { Liners } \\
\text { Shippers }\end{array}$ & $0.003 *$ & $\begin{array}{c}0.980 \\
0.021 *\end{array}$ \\
\hline Shipping line operation & $\begin{array}{l}\text { Liners } \\
\text { Shippers }\end{array}$ & 0.061 & $\begin{array}{c}0.030^{*} \\
0.931\end{array}$ \\
\hline Terminal operation & $\begin{array}{l}\text { Liners } \\
\text { Shippers }\end{array}$ & $0.050^{*}$ & $\begin{array}{l}0.495 \\
0.582\end{array}$ \\
\hline Transportation Distance & $\begin{array}{l}\text { Liners } \\
\text { Shippers }\end{array}$ & 0.602 & $\begin{array}{l}0.699 \\
0.995\end{array}$ \\
\hline Intermodal System & $\begin{array}{l}\text { Liners } \\
\text { Shippers }\end{array}$ & 0.536 & $\begin{array}{l}0.712 \\
0.977\end{array}$ \\
\hline
\end{tabular}

Note: * Mean differences are significant at $\mathrm{P}<0.05$

The empirical results show significant perspective differences among maritime/port market players regarding the following three factors: 'hinterland and terminal basic condition', 'shipping line operation', and 'terminal operation'. Regarding 'hinterland and terminal basic condition', shipping lines and terminal operators have a similar perspective and impose more weight compared to shippers. For 'shipping line operation', liners impose a significant importance while shippers and terminal operators give less weight. In addition, liners and terminal operators give more significance to 'terminal operation' factor than shippers.

\section{Implication and Conclusions}

The current empirical research shows that significant differences among the market players exist, especially regarding three factors: 'hinterland and terminal basic condition', 'shipping liners' operations' and 'terminal operations'. First, liners and terminal operators 
give more weights on hinterland and terminal basic condition such as cargo volume, market size, port depth, nearness to hinterland and terminal handling charge (THC), and berth availability than shippers. Second, terminal operators do not seriously considering line operation involving the attributes of liners' schedule reliability and service frequency, variety of routes, direct calling, alliance members or competitors calling, and balanced cargo, compared to carriers and shippers. In addition, terminal operation factors such as accessibility, port size, infrastructure, feeder network, and port tariffs are more critically considered by liners and terminal operators than by shippers.

These findings indicate that liners and terminal operators place considerable importance on hinterland condition and terminal function/operations, while shippers have no specific interest in these factors. These results imply that shippers are primarily concerned with getting a shipment from its origin to its destination, paying little attention to the intermediary points through which it is moved (Malchow and Kanafani, 2001). In addition, the current study shows that shippers and liners possess similar perspectives for the importance of liners' operations in a port, which is not considered too highly by terminal operators. Therefore, it is recommended that terminal operators make more effort to reinforce their capabilities accommodating and supporting liners' operations and strategies in order to obtain and maintain their competitive advantage and position. In conclusion, ports, particularly terminal operators, should focus their attention on the factors on which shippers and liners commonly place high priority, when they formulate, implement and evaluate their terminal management policiesy, operating strategies and marketing plans.

The current study provides a comprehensive understanding of the different market players' heterogeneous criteria adopted when they determine calling ports and design service routes or establish port development plans and implement terminal operation strategies by utilizing critical port selection attributes and factors, and utilizing a series of rigorous statistical methods. An idea for a meaningful future study would be to test the explanation power of the port choice factors and real influences of major market players on port selection factors. For instance, it would be possible to collect the scores of various container terminals for each port selection factor and design a regression model examining the influences of these factors upon ports' market shares and/or throughputs. In addition, a structural equation modeling could be employed to explore significant direct and indirect relationships among port selection factors and port operational indices. 


\section{Acknowledgements}

This work was supported by INHA UNIVERSITY research grant.

\section{References}

Bird, J. and Bland, G. (2002) Freight Forwarders Speak, Maritime Policy and Management, vol.15, no.1, pp. 35-55.

Chang, Y. T., Lee, S. Y. and Lie, S. G. (2002) Factors Affecting Liners' Port Selection by Trade Route, Korea Maritime Institute.

D'Este, G. M. and Meyrick, S. (1992) Carrier selection in a RO/RO ferry trade Part 1 Decision factors and attitudes, Maritime Policy and Management, vol.19, no2, pp.115-126.

De Langen, PW. (2007), Port competition and selection in contestable hinterlands: the case of Austria, European Journal of Transport and Infrastructure Research, Vol.7, No.1, pp.1-14.

Dillion, W. and Goldstein, M. (1984) Multivariate Analysis: Methods and Applications, N.Y., John Wiley \& Sons.

Douglas, K. and Fleming, A. (2000) Geographical Perspective of the Transshipment Function, International Journal of Maritime Economics, Vol.2, No.3, pp.163-176.

Fleming, D. K. (1997) World container port rankings, Maritime Policy and Management, Vol.24, No.2, pp.175-181.

Gibson, B, Sink, H. and Mundy, R. (1993) Shipper-Carrier Relationship and Carrier Selection Criteria, Logistics and Transportation Review, Vol.29, No.4, pp. 371-382.

Hayuth, Y. (1987) Intermodality: Concept and Practice, London, U.K.: Lloyd's of London Press. Hayuth, Y. and Fleming, D. K. (1994) Concepts of Strategical Commercial Location: the Case of Container Ports, Maritime Policy and Management, Vol.21, No.3, pp.187-193.

Lago, A., Malchow, M. and Kanafani, A. (2001) An Analysis of Carriers' Schedules and the Impact on Port Selection, Proceedings of IAME Annual Conference 2001, pp. 123-137.

Lirn, T. C., Thanopoulou, H. A, Beynon, M. J. and Beresford, A. K. C. (2004) An Application of AHP on Transhipment Port Selection: A Global Perspective, Maritime Economics \& Logistics, Vol.6, pp.70-91.

Lu, C. (2000) Logistics Services in Taiwanese Maritime Firms, Transportation Research Part E, vol. 36, pp. 79-96. 
Malchow, M. and Kanafani, A. (2001) A disaggregate analysis of factors influencing port selection, Maritime Policy and Management, Vol.28, No.3, pp.265-277.

Malchow, M. and Kanafani, A. (2004) A disaggregate analysis of port selection, Transportation Research Part E, Vol.40, pp.317-337.

Murphy, P., Daley, J. and Dalenberg, D. (1992) Port selection criteria: an application of a transport research framework, Logistics and Transportation Review, Vol.28, No.3, pp.237-255.

Murphy, P. and Daley, J. (1994) A Comparative Analysis of Port Selection Factors, Transportation Journal, Vol.34, No.1, pp. 15-21.

Nir, A., Lin, K. and Liang, G. (2003) Port Choice Behavior- from the Perspective of the Shipper, Maritime Policy and Management, Vol.30, No.2, pp. 165-173.

Slack, B. (1985) Containerisation and inter-port competition, Maritime Policy and Management, Vol.12, No.4, pp.293-304.

Song, D-W. Yeo, K-T. (2004) A competitive Analysis of Chinese Container Ports Using the Analytic Hierarchy Process, Maritime Economics \& Logistics, Vol.6, pp.34-52.

Tiwary, P, Itoh, H. and Doi, M. (2004) Shippers' Port and Carrier Selection Behaviour in China: A Discrete Choice Analysis, Maritime Economics \& Logistics, Vol.5, pp.23-39.

Tongzon, J. L. and Sawant, L. (2007), Port Choice in a Competitive Environment: From the Shipping Lines' Perspective, Applied Economics, Vol. 39, pp.477-492.

Zan, Y. (1999) Analysis of Container Port Policy by the Reaction of an Equilibrium Shipping Market, Maritime Policy and Management, Vol.26, No.4, pp. 369-381. 